\title{
Controlled releasing properties of gelatin nanofabric device containing chlorhexidine
}

\author{
Dae-Ung Park ${ }^{1}$, Heung-Sik Um² , Beom-Seok Chang ${ }^{2}$, Si-Young Lee ${ }^{3}$, Ki-Yeon Yoo ${ }^{4,5}$, Won-Youl Choi ${ }^{5,6,7 *}$, and Jae-Kwan Lee ${ }^{5,8 *}$
}

\begin{abstract}
${ }^{1}$ Master's Student, Department of Periodontology, Gangneung-Wonju National University, Gangneung, Republic of Korea ${ }^{2}$ Professor, Department of Periodontology, Gangneung-Wonju National University, Gangneung, Republic of Korea ${ }^{3}$ Professor, Department of Microbiology and Immunology, Gangneung-Wonju National University, Gangneung, Republic of Korea ${ }^{4}$ Professor, Department of Anatomy, Gangneung-Wonju National University, Gangneung, Republic of Korea

${ }^{5}$ Professor, Research Institute for Dental Engineering, Gangneung-Wonju National University, Gangneung, Republic of Korea ${ }^{6}$ Professor, Department of Metal and Materials Engineering, Gangneung-Wonju National University, Gangneung, Republic of Korea ${ }^{7} \mathrm{CEO}$, Wellnanos Co., Ltd., Gangneung, Republic of Korea

${ }^{8}$ Professor, Department of Periodontology, Gangneung-Wonju National University, Gangneung, Republic of Korea
\end{abstract}

We aimed to evaluate the degradation and antimicrobial activity of novel chlorhexidine-containing gelatin nanofabric devices and their cytotoxicity in animals. The electro-spun device with a size of $3 \times 4 \times 0.4 \mathrm{~mm}^{3}$ was prepared by entrapping chlorhexidine gluconate (CHX) in a gelatin matrix. The devices were divided into three groups based on the CHX percentage (G1: 46\%, G2: 50\%, and G3: 54\%), and the commercial product, PerioChip (CP), was used for the control group. We used an in vitro test for the degradation and antibacterial activity and investigated cytotoxicity using an in vivo test. Artificial saliva was used for the degradation test of chips and blood agar plates seeded with the oral bacteria Streptococcus sanguinis to address antibacterial activity. Furthermore, the devices were inserted between the skin and muscle of rat abdomens to evaluate infection and inflammatory reactions. We detected no obvious differences in the antibacterial or degradation test results between the test and control groups. In the histological analysis, the scaffold without CHX showed no signs of infection or inflammatory reaction. Both CHX-containing groups exhibited inflammation and necrosis in the muscle and skin, although the reaction in the test group was milder. In the degradation tests, antibacterial tests, and cytotoxic reactions in animal experiments, the electro-spun gelatin-based device showed similar results to those of the commercial device in the control group. Further studies on the quantitative analysis and clinical outcome evaluation of the electro-spun gelatin-based device in humans are necessary.

Key Words: Anti-bacterial agents; Chlorhexidine; Drug delivery systems; Periodontal pocket

(c) This is an open-access article distributed under the terms of the Creative Commons Attribution Non-Commercial License (http://creativecommons.org/licenses/by-nc/4.0) which permits unrestricted noncommercial use, distribution, and reproduction in any medium, provided the original work is properly cited.

Received March 18, 2021; Revised April 29, 2021; Accepted May 13, 2021

${ }^{\star}$ Co-Corresponding author: Jae-Kwan Lee, Department of Periodontology, Gangneung-Wonju National University College of Dentistry, 7 Jukheon-gil, Gangneung 25457, Republic of Korea.

Tel: +82-33-640-3199, Fax: +82-33-640-3113, E-mail: periojk@gwnu.ac.kr

*Co-Corresponding author: Won-Youl Choi, Department of Metal and Materials Engineering, Gangneung-Wonju National University, 7 Jukheon-gil, Gangneung 25457, Republic of Korea.

Tel: +82-33-640-2483, Fax: +82-33-640-3113, E-mail: cwy@gwnu.ac.kr

Copyright $\odot$ 2021, Oral Biology Research Institute 


\section{Introduction}

A deep periodontal pocket provides an anaerobic environment in which pathogenic bacteria can grow and proliferate [1]. The interaction of the complex subgingival bacterial population with the host immune response causes destruction of the periodontium. Suspected pathogens have been shown to secrete active molecules that directly act on host tissues. The consequent production of various inflammatory and immune mediators by the host may cause additional tissue destruction [2]. Mechanical debridement is the most effective and commonly used method used to remove dental biofilms. However, mechanical debridement does not eliminate all pathogens from deep pockets; it only decreases the bacterial load. In fact, periodontal pathogens exist within periodontal pockets or in areas inaccessible to instrumentation [3]. Therefore, adjunctive treatment to improve the efficacy of mechanical debridement, such as systemic/local antibiotics, or antimicrobial photodynamic therapy, have been proposed [4-6].

Administration of systemic antibiotics is the primary antimicrobial strategy for the elimination of microflora living in periodontal pockets. However, it is hard to attain the necessary antibiotic concentration in periodontal pockets. Also, administration of systemic antibiotics may cause adverse effects, such as drug toxicity, antibiotic resistance, and drug interactions. In particular, the long-term use of systemic antibiotics is associated with a potential risk of producing resistant bacteria and superimposed bacterial infections $[4,7]$. Because systemic antibiotics for periodontal therapy has drawbacks, local drug delivery systems for antimicrobial agents have been designed to administer antimicrobial agents directly to periodontal pockets. By concentrating the drug at its target site to achieve high concentrations, a local drug delivery system avoids the adverse effects associated with systemic antibiotic administration [8]. For the successful use of these controlled delivery devices, it is crucial to combine a scaffold for constant delivery of drugs with the delivery of adequate concentrations of active agents to the pocket.

Local drug delivery systems can be categorized into two groups depending on whether they degrade: nondegradable materials and degradable materials. Non- degradable scaffolds have the advantage that the clinician can determine the timing of removal. However, there are disadvantages that a second visit is required for removal and have the possibility of undesirable effects when they are left in the periodontal pockets for too long. Degradable scaffolds have the advantage that they only require a single visit for the insertion of the device. Various types of local drug delivery systems used in the treatment of periodontal diseases and peri-implant diseases include fibers, film, injectable systems, gels, strips, compacts, vesicular systems, microparticle systems, nanoparticle systems, and nanotube systems [9-12].

Gelatin is a candidate for the scaffold of a controlled local drug delivery device. Gelatin is a natural protein derived from the hydrolysis of collagen and is highly biocompatible and biodegradable in a physiological environment [13]. Even though gelatin is commonly derived from animals, gelatin exhibits very low antigenicity from the formation of nontoxic metabolites during the degradation process [14]. Because gelatin is inexpensive and highly available, it is widely used in pharmaceutical and medical applications. In addition, it is regarded as a generally recognized as safe substance by the United States Food and Drug Administration (FDA) [15].

Several studies related to local delivery devices using gelatin-based materials for treating periodontal diseases have been described [16,17]. In this study, to control the drug delivery properties, the gelatin structure was designed as a nanofiber structure containing chlorhexidine (CHX) instead of a scaffold structure in which CHX is coated on it. Without the CHX coating process, the gelatin nanofibers containing CHX were simultaneously fabricated by the electro-spinning process using precursors including gelatin, CHX gluconate, and glutaraldehyde. The gelatin devices were molded with gelatin nanofibers with various amounts of CHX. The characteristics of gelatin devices that can be degradable and sustained elution were evaluated using an in vitro test and their cytotoxicity was investigated by an in vivo test as part of the process of selecting the best formulation for future clinical testing. 


\section{Materials and Methods}

\section{In vitro test}

\section{Device preparation}

Gelatin devices containing 20\% CHX gluconate (SigmaAldrich, Darmstadt, Germany) were fabricated using an electro-spinning and forming process. The devices were composed of a gelatin fiber matrix with a several hundred nanometer diameters. The gelatin fiber matrix was composed of gelatin, CHX gluconate, and glutaraldehyde. To observe the effects of CHX gluconate in the device, various amounts of $20 \%$ CHX gluconate such as $46 \%, 50 \%$, and $54 \%$ were chosen and named G1, G2, and G3 groups, respectively, for this study. To fabricate the G1, G2, and G3 group, various volumes of 20\% CHX gluconate such as 46\%, 50\%, and $54 \%$ were measured and put it in the gelatin precursor for the electro-spinning process. The precursors were electrospun to obtain the gelatin fiber matrix. The shape of the devices was optimized for insertion into the periodontal pocket. The size of the devices was $3 \times 4 \times 0.4 \mathrm{~mm}^{3}$ and the actual devices are shown in Fig. 1.

\section{Device degradation}

The devices and control specimens (PerioChip, CP; Perio Products, Ltd., Jerusalem, Israel) were submerged in an Eppendorf tube with $1 \mathrm{~mL}$ of artificial saliva (DDW 40\%,

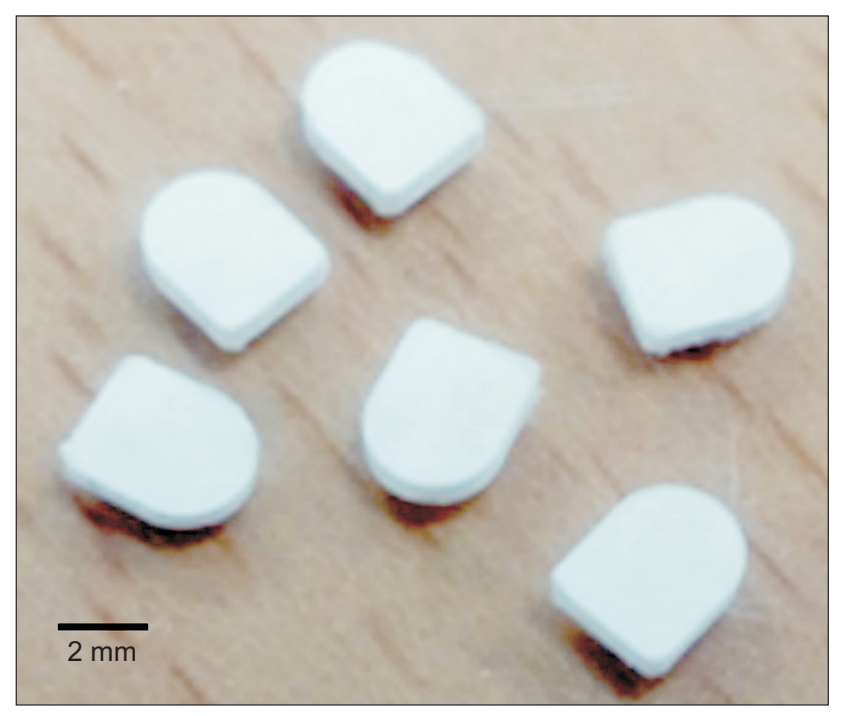

Fig. 1. The gelatin devices fabricated by electrospinning method. concentrated glycerin 10\%, sodium carboxyl methyl cellulose $50 \%$ at $37^{\circ} \mathrm{C}$ in a shaking water bath (BS-30; Jeio Tech, Seoul, Korea) at $100 \mathrm{rpm}$. To monitor the degradation of the devices under conditions similar to the oral environment, a flow cell test was used with an artificial saliva having a flow rate of $8.2 \mathrm{~mL} / \mathrm{min}$ into the Eppendorf tube. This procedure was continued for 10 days and the degradation of the devices was observed by visual inspection [18]. All experiments were repeated at least twice.

\section{Antibacterial tests}

Devices, cut into square shapes with two round edges, and control specimens (PerioChip, CP; Perio Products, Ltd.) were placed on blood agar plates (Hanil-KOMED, Seongnam, Korea) seeded with the oral bacteria, Streptococcus sanguinis ATCC 10556 (Department of Microbiology and Immunology, College of Dentistry, Gangneung-Wonju National University). After 24 hours of incubation at $37^{\circ} \mathrm{C}$ in an aerobic environment supplemented with $5 \% \mathrm{CO}_{2}$, the devices were transported to newly seeded blood agar plates for an additional 24 hours of incubation. This procedure was repeated for 28 days. The growth inhibition area of the bacteria on the agar plate was measured using a computer program, Image J (National Institutes of Health, Bethesda MD, USA), to calculate the area of inhibition around the device.

\section{In vivo test}

\section{Animals}

Male Sprague-Dawley rats, weighing 200-250 g, were anesthetized using injections of $1.0 \mathrm{~mL} / 100 \mathrm{~g}$ body weight of a solution containing sodium pentobarbital $(60 \mathrm{mg} / \mathrm{mL})$ and $\mathrm{NaCl}(9 \mathrm{mg} / \mathrm{mL})$ in 1:9 volume proportions. The experimental protocol was approved by the Animal Ethics Committee of Gangneung-Wonju National University College of Dentistry (GWNU-2016-33), South Korea.

\section{Implantation procedure}

The test and control samples were implanted in rat abdominal walls. The implantation procedure was performed as described previously by Rosengren et al. [19]. The abdominal muscle sheath was opened and the muscle put 
aside. The devices (G1, G2, and G3), control specimens (PerioChip; Perio Products, Ltd.), and negative control devices (non-CHD), which did not contain CHX in the gelatin matrix, were inserted on the linea alba, outside the peritoneum. The abdominal muscle was put back to cover the devices and sutures were placed in the muscle sheath. There were five animals in each of the five groups.

\section{Implant retrieval and histology}

The animals were sacrificed after one week. The tissue surrounding the implants was detached en bloc by removing a section of the peritoneal membrane without injuring the specimen. Damage to soft tissue was observed by visual inspection.

The detached tissues were fixed in $4 \%$ buffered formalin for 24 hours. After that, the specimens were dehydrated in an ascending series of ethanol solutions, embedded in paraffin, and stained with hematoxylin \& eosin using Masson's trichrome method.

\section{Image processing}

All images were obtained using a digital camera (A6000; Sony, Tokyo, Japan) and microscope (Axio Imager A2; Zeiss, Jena, Germany), using bright field illumination.

\section{Results}

\section{Degradation test}

During the degradation period of 10 days, the devices were very slowly melted away and the concentration of CHX in total artificial salvia was $6.50 \times 10^{-6} \mathrm{mg} / \mathrm{mL}$. Fig. 2 shows the devices of G1, G2, G3, and CP in the Eppendorf tube for 10 days. It is observed that the G1 and CP groups are more degraded than other groups, but noticeable differences were not observed. The devices of all the groups were not degraded completely for 10 days. The matrices of the device were more quickly degraded in the early stage than in the late stage.

\section{In vitro antibacterial test}

Fig. 3 shows antibacterial profiles of G1, G2, G3, and CP with the oral bacteria Streptococcus sanguinis obtained by the disc diffusion test. Similar antibacterial profiles were shown in G1, G2, and G3 devices. All groups exhibited antibacterial activity over a period of 28 days. The antibacterial activity of the $\mathrm{CP}$ group was much wider than that of the G1, G2, and G3 groups in the early stages. However, the G1, G2, G3, and CP groups displayed similar antibacterial activity in the late stages. The areas of inhibition zone of all groups on Streptococcus sanguinis were measured and summarized in Table 1.

\section{In vivo cytotoxicity test}

To consider the cytotoxicity of the gelatin nanofabric matrix without chlorhexidine, non-CHD group was tested instead of G1 group. Fig. 4 shows the abdominal wall of Male Sprague-Dawley rats after implantation of non-CHD, CP, G2, and G3. The control in Fig. 4 means the abdominal wall of Male Sprague-Dawley rats without the device. The non-CHD group displayed a clinical sign of infection or inflammatory reaction. No residual gelatin devices were seen in the non-CHD group. In the CP group, necrotizing tissue and scars were found in the outer space of the abdominal wall. Also, residual devices and hyperemia were found in the inner space of the abdominal wall, in the muscle layer.

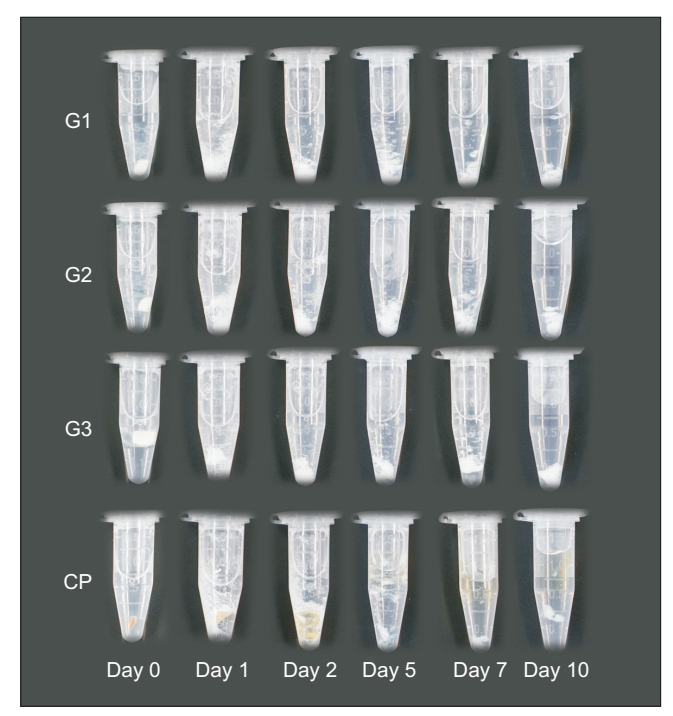

Fig. 2. G1, G2, G3, and CP devices in an Eppendorf tube for 10-day degradation test. G1 and CP groups look like more degraded than other groups, but no noticeable difference is observed. CP, PerioChip. 

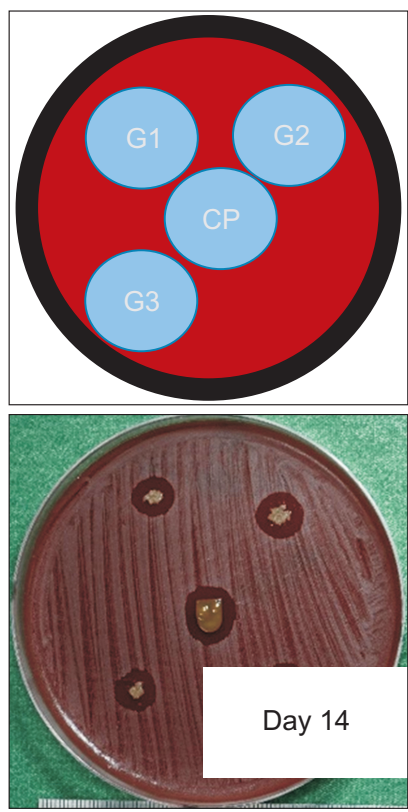

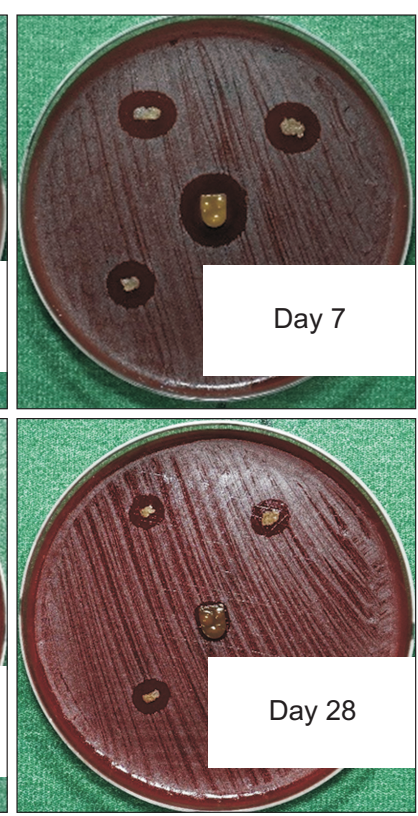

Fig. 3. G1, G2, G3, and CP devices on blood agar plates for the antibacterial test with Streptococcus sanguinis. All groups exhibited antibacterial activity over a period of 28 days. In the early stages, the antibacterial activity of the $\mathrm{CP}$ group was much wider than other groups, however, all groups displayed similar antibacterial activity in the late stages. CP, PerioChip.

Table 1. Area of inhibition zones obtained by antibacterial test

\begin{tabular}{ccccc}
\hline & \multicolumn{3}{c}{ Groups } \\
\cline { 2 - 5 } Days & G1 & G2 & G3 & CP \\
\hline Day 2 & $202(100)$ & $218(100)$ & $165(100)$ & $278(100)$ \\
Day 7 & $127(62)$ & $120(54)$ & $94(57)$ & $231(82)$ \\
Day 14 & $67(33)$ & $92(42)$ & $68(41)$ & $126(45)$ \\
Day 21 & $66(32)$ & $79(36)$ & $65(39)$ & $90(32)$ \\
Day 28 & $55(27)$ & $63(28)$ & $54(33)$ & $59(21)$ \\
\hline
\end{tabular}

Values are presented as number (\%). Area of inhibition zone (unit: $\mathrm{mm}^{3}$ ).

CP, PerioChip.

Similar, but milder, histological results were seen in the G2 and G3 groups.

Fig. 5 shows the histopathology of the cross-sectional specimen obtained by hematoxylin \& eosin and Masson' $s$ trichrome staining. In the control group, all of layers in the abdominal wall were normal. In the non-CHD group, any inflammatory or necrotic tissues were not found. However, the abdominal wall tissue of the $\mathrm{CP}$ group shows not only extensive cell necrosis in the epidermis, dermis and hypodermis layers but also destruction of muscle fibers in abdominal muscles. Furthermore, the inflammatory cells in this lesion were not observed due to severe necrotic response. The destructions of muscle fibers were marked with white arrows in Fig. 5E and F. In G2 and G3 groups, necrotic, inflammatory tissue and destruction of muscle fiber which is similar to $\mathrm{CP}$ group were also found but the lesion was less than CP group. The sign of inflammation was also marked with asterisks.

\section{Discussion}

In this study, we investigated a new gelatin nanofabric device to control the release of $\mathrm{CHX}$ with physiologic properties and cytotoxicity in animals that were similar to those associated with PerioDevice, a commercially available product for the treatment of deep periodontal pockets.

Steinberg et al. [20]. have stated that the protein crosslinking reaction allowed for controlled release and slow degradation with this type of device. In addition, they found that the higher the degree of cross-linking, the slow- 


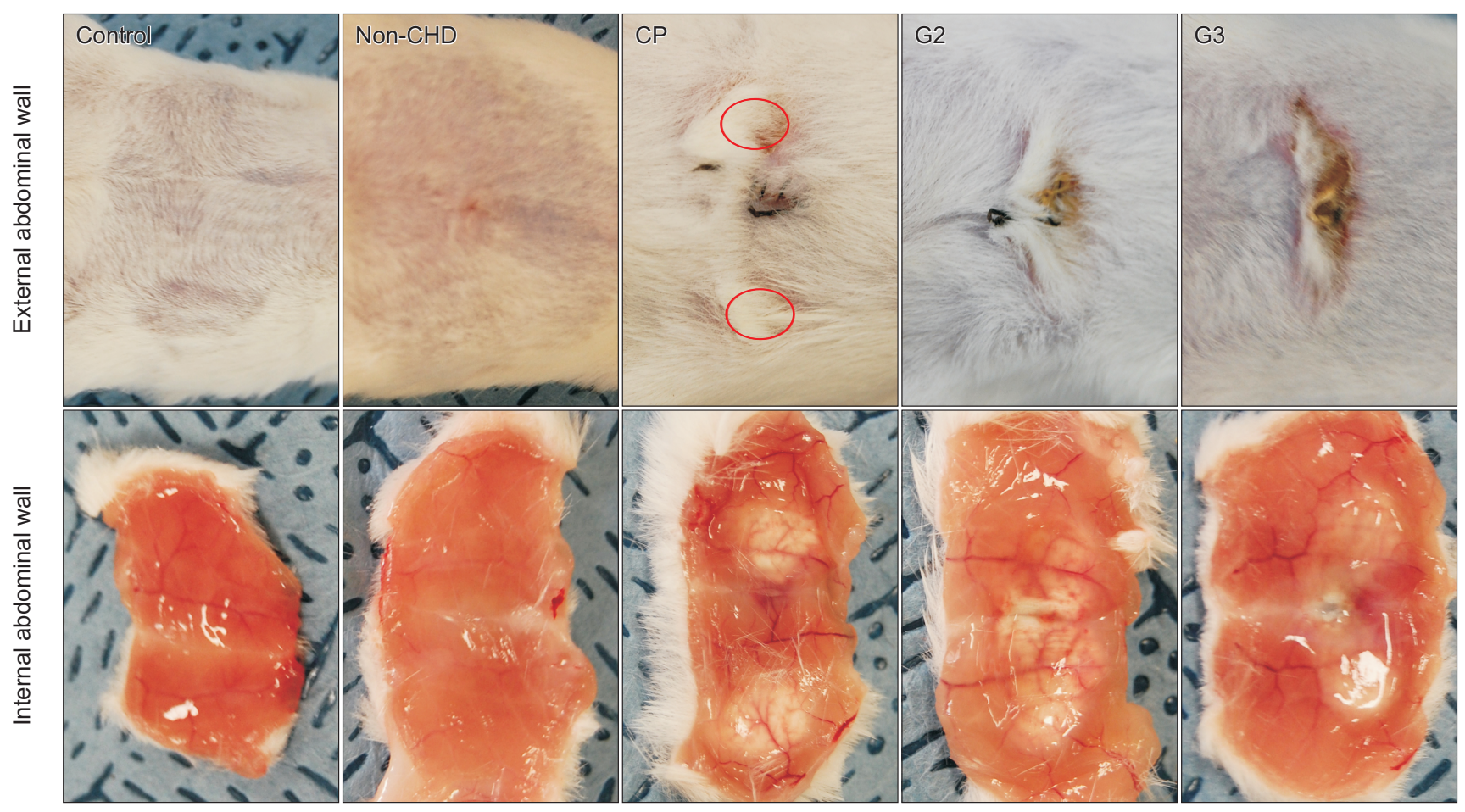

Fig. 4. The abdominal wall of male Sprague-Dawley rats after implantation of non-CHD, CP, G2, and G3. The control group and non-CHD groups showed no sign of inflammation or necrosis. However, the CP, G2, and G3 groups exhibited necrosis and scars in the outer spaces of the abdominal wall (circle). CP, PerioChip; non-CHD, negative control devices.

er the rate of drug release and device degradation and the longer the duration of antibacterial activity. In this study, the cross-linking reaction was caused by glutaraldehyde, which allowed the device to possess a controlled-release physiologic property that was useful for therapeutic purposes. CHX is also known to coact chemically with proteins [21] and this coaction plays a role in the controlledrelease mechanism of CHX from the devices and the rate of device degradation. This study indicated that incorporation of CHX into the device did not eliminate the antibacterial activity of CHX or change the rate of degradation of the device.

It is considered most important in devising a sustained release device that the delivery of effective amounts of antibacterial agents is sustained for a length of time sufficient to affect deep periodontal pocket microflora. In vivo studies are being carried out with various formulations to select a formulation that fulfills the above criteria for clinical testing in the treatment of periodontal diseases. In this study, there were no obvious differences between the G1, G2, or G3 groups in the degradation test or the antibacterial test.
Previous studies indicated that the duration of release of CHX determined the efficacy of CHX on the deep sulcus flora. Intra-sulcular irrigation of periodontal pockets with CHX had only a short persistent effect on the deep periodontal pocket microflora. However, a sustained release of CHX over 3 days resulted in a longer duration of effect and significant changes for up to 14 days, whereas 9 days of sustained exposure to CHX resulted in significant changes for as long as 11 weeks [22-24]. In this study, the gelatin nanofabric device containing CHX was maintained for 10 days in a degradation test and for 28 days in an antibacterial test, results similar to those of PerioDevice. By analogy with the result of this study, the gelatin nanofabric device containing CHX should be effective for deep periodontal pockets owing to the sustained release of CHX over a long duration.

A previous multi-center study by Soskolne et al. [25]. have stated that PerioDevice was an effective adjunctive treatment along with scaling and root planing (SRP) for the treatment of chronic periodontitis patients. SRP and this adjunctive treatment provided significantly greater reduc- 


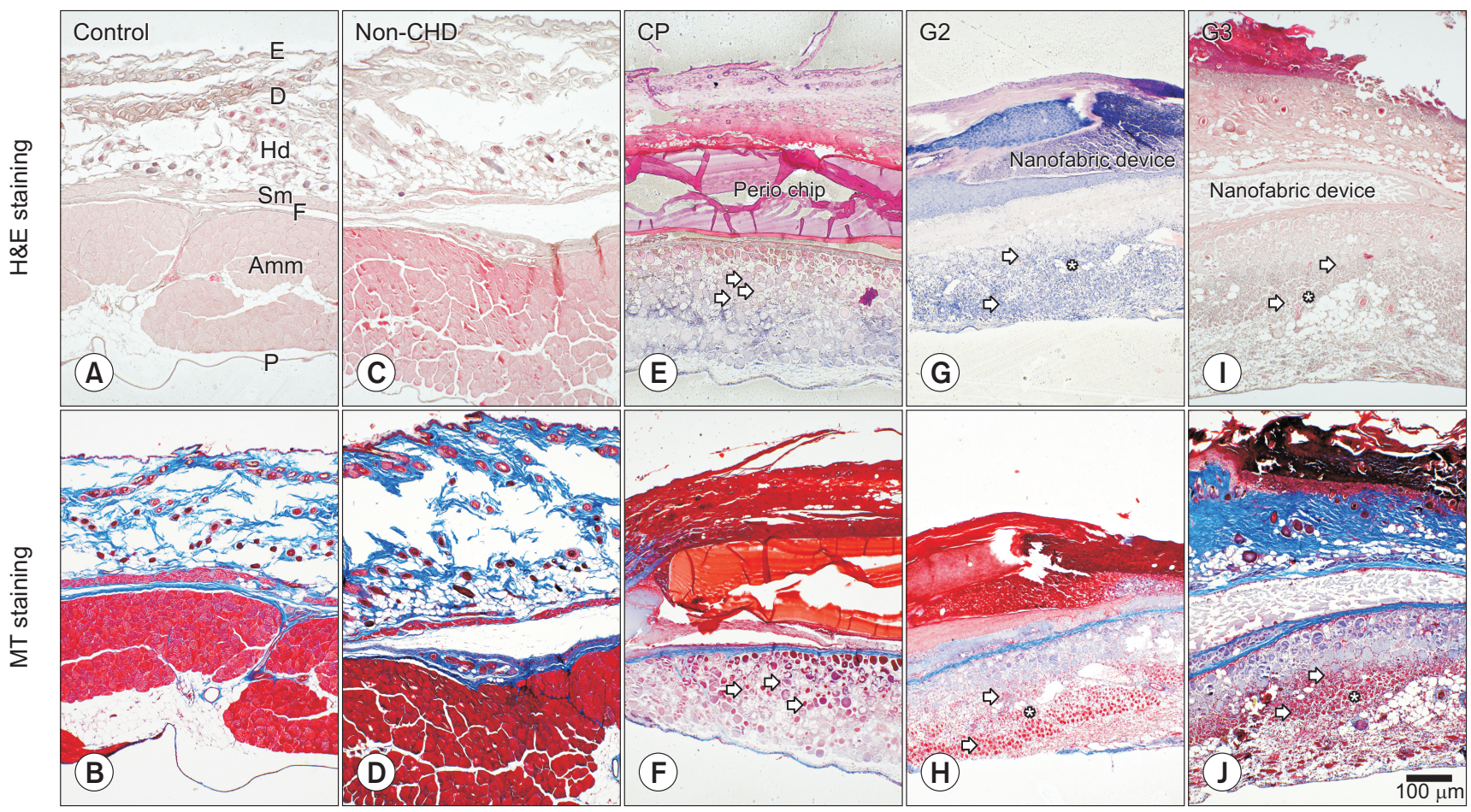

Fig. 5. Tissue was stained with hematoxylin \& eosin using Masson's trichrome method. The control group (A, B) and non-CHD group (C, D) displayed no abnormal signs. The CP (E, F), G2 (G, H), and G3 (I, J) groups displayed necrosis of muscles, residual chips, and signs of inflammation. However, the G2 and G3 groups displayed a milder reaction. E, epidermis; D, dermis; Hd, hypodermis; Sm, subcutaneous muscle; F, fascia; Amm, abdominal muscles; P, peritoneum; white arrow, destruction of muscle fibers; asterisk, signs of inflammation; CP, PerioChip; non-CHD, negative control devices.

tions in periodontal pocket depth (PPD) at 6 months than SRP alone. A similar result was shown in the study of Jeffcoat et al. [26]. where SRP and this type of adjunctive treatment showed a significantly greater reduction in PPD at both 6 and 9 months than SRP alone. In a study by Pattnaik et al. [27], another local drug delivery system, PerioCol CG (Eucare Pharmaceuticals, Chennai, India), exhibited good clinical outcomes in sites that responded poorly to SRP alone.

CHX has been widely used as a topical antiseptic. CHX digluconate (4.0\%) was approved by the FDA as a commercial product [28]. In addition, the FDA approved the medicinal use of a mouth rinse containing $0.12 \% \mathrm{CHX}$ gluconate that can be used over the long-term [29]. Harvey et al. [30] have stated that CHX digluconate caused white soft tissue lesions and hyperplasia in hamster cheek pouch tests when it was applied topically at a concentration of $2.0 \%$. PerioDevice has been safely used for patients with periodontitis who cannot be treated with SRP alone. In this study, there was no irritation or tissue damage in the nonCHD group, which meant that the gelatin matrix was stable and safe to use in animals. This study also indicated that all devices containing CHX caused tissue necrosis and scars in histological analysis. However, the G1, G2, and G3 groups displayed milder reactions than the control group. This may indicate that the gelatin nanofabric device containing CHX could be safely used.

One limitation of the present study was that the in vitro test result was evaluated only by visual inspection, which meant that this study provided only incomplete information. There are a few methods that are useful for quantitatively analyzing CHX in solution, including ultraviolet spectrometry and high performance liquid chromatography [31]. However, there is no optimized protocol for analyzing CHX in artificial saliva.

Another limitation is that artificial saliva has no enzymes like those that are found in gingival crevicular fluid. The results of a degradation test using artificial saliva and one 
using gingival crevicular fluid may not agree. However, it is very hard to get enough gingival crevicular fluid to perform an in vitro test. Therefore, quantitative analysis is needed from human studies.

Stanley et al. [32] have reported that $125 \mu \mathrm{g} / \mathrm{mL}$ of CHX was inhibitory to $99 \%$ of bacterial isolated from periodontal pockets. Therefore, the gelatin nanofabric device containing CHX should allow the CHX concentration to reach this critical concentration in gingival crevicular fluid.

The gelatin nanofabric device containing CHX had physiologic properties similar to those of PerioDevice regarding degradation and antibacterial ability. The gelatin nanofabric device also exhibited cytotoxic reaction similar to that of PerioDevice in animal experiments. The gelatin nanofabric device without CHX displayed no sign of infection or inflammatory reaction. The gelatin nanofabric device containing CHX will be very useful for the treatment of chronic periodontitis patients and human study is needed for quantitative analysis and clinical outcome evaluation.

\section{Acknowledgements}

This study was financially supported by Research and Development Program of the Korea Industrial Complex Corp. through the Ministry of Trade, Industry and Energy (Grant No. RDNWGW1507) and National Research Foundation of Korea (Grant No. 2019R1I1A3A01057765).

\section{Conflicts of Interest}

The authors declare that they have no competing interests.

\section{ORCID}

$$
\begin{aligned}
& \text { Dae-Ung Park } \\
& \text { https://orcid.org/0000-0003-1617-9888 } \\
& \text { Heung-Sik Um } \\
& \text { https://orcid.org/0000-0002-7986-1019 } \\
& \text { Beom-Seok Chang } \\
& \text { https://orcid.org/0000-0002-5280-3249 } \\
& \text { Si-Young Lee } \\
& \text { https://orcid.org/0000-0001-8826-1413 }
\end{aligned}
$$

\author{
Ki-Yeon Yoo \\ https://orcid.org/0000-0002-0575-275X \\ Won-Youl Choi \\ https://orcid.org/0000-0002-2181-827X \\ Jae-Kwan Lee \\ https://orcid.org/0000-0003-1710-1580
}

\section{References}

1. Marsh PD. Host defenses and microbial homeostasis: role of microbial interactions. J Dent Res 1989;68:1567-1575.

2. Meyle J, Chapple I. Molecular aspects of the pathogenesis of periodontitis. Periodontol 2000 2015;69:7-17. doi: 10.1111/prd.12104.

3. Haffajee AD, Cugini MA, Dibart S, Smith C, Kent RL Jr, Socransky SS. The effect of SRP on the clinical and microbiological parameters of periodontal diseases. J Clin Periodontol 1997;24:324-334. doi: 10.1111/j.1600-051x.1997. tb00765.x.

4. Heitz-Mayfield LJ. Systemic antibiotics in periodontal therapy. Aust Dent J 2009;54 Suppl 1:S96-S101. doi: 10.1111/ j.1834-7819.2009.01147.x.

5. Mombelli A, Samaranayake LP. Topical and systemic antibiotics in the management of periodontal diseases. Int Dent $J$ 2004;54:3-14. doi: 10.1111/j.1875-595x.2004.tb00246.x.

6. Ahad A, Lamba AK, Faraz F, Tandon S, Chawla K, Yadav N. Effect of antimicrobial photodynamic therapy as an adjunct to nonsurgical treatment of deep periodontal pockets: a clinical study. J Lasers Med Sci 2016;7:220-226. doi: 10.15171/jlms.2016.39.

7. Ardila CM, Granada MI, Guzmán IC. Antibiotic resistance of subgingival species in chronic periodontitis patients. J Periodontal Res 2010;45:557-563. doi: 10.1111/j.16000765.2010.01274.x.

8. Goodson JM, Hogan PE, Dunham SL. Clinical responses following periodontal treatment by local drug delivery. J Periodontol 1985;56 Suppl 11S:81-87. doi: 10.1902/ jop.1985.56.11s.81.

9. Garg S. Local drug delivery systems as an adjunct to cure periodontitis- the novel dental applicant. Pharm Methods 2015;6:1-8.

10. H R R, Dhamecha D, Jagwani S, Rao M, Jadhav K, Shaikh S, Puzhankara L, Jalalpure S. Local drug delivery systems in the management of periodontitis: a scientific review. J Control Release 2019;307:393-409. doi: 10.1016/ j.jconrel.2019.06.038.

11. Kaplish V, Walia MK, Hari Kumar H. Local drug delivery systems in the treatment of periodontitis: a review. Pharmacophore 2013;4:39-49.

12. Lee JK, Choi DS, Jang I, Choi WY. Improved osseointegration of dental titanium implants by $\mathrm{TiO} 2$ nanotube arrays 
with recombinant human bone morphogenetic protein-2: a pilot in vivo study. Int J Nanomedicine 2015;10:11451154. doi: 10.2147/IJN.S78138.

13. Nezhadi SH, Choong PF, Lotfipour F, Dass CR. Gelatinbased delivery systems for cancer gene therapy. J Drug Target 2009;17:731-738. doi: 10.3109/10611860903096540.

14. Olsen D, Yang C, Bodo M, Chang R, Leigh S, Baez J, Carmichael D, Perälä M, Hämäläinen ER, Jarvinen M, Polarek J. Recombinant collagen and gelatin for drug delivery. Adv Drug Deliv Rev 2003;55:1547-1567. doi: 10.1016/ j.addr.2003.08.008.

15. Elzoghby AO. Gelatin-based nanoparticles as drug and gene delivery systems: reviewing three decades of research. J Control Release 2013;172:1075-1091. doi: 10.1016/ j.jconrel.2013.09.019.

16. Karthikeyan K, Durgadevi R, Saravanan K, Shivsankar K, Usha S, Saravanan M. Formulation of bioadhesive carbomer gel incorporating drug-loaded gelatin microspheres for periodontal therapy. Trop J Pharm Res 2012;11:335-343. doi: 10.4314/tjpr.v11i3.1.

17. Madhumathi K, Jeevana Rekha L, Sampath Kumar TS. Tailoring antibiotic release for the treatment of periodontal infrabony defects using bioactive gelatin-alginate/apatite nanocomposite films. J Drug Deliv Sci Technol 2018;43:5764. doi: 10.1016/j.jddst.2017.09.015.

18. Kothari S, Gnanaranjan G, Kothiyal P. Periodontal chip: an adjunct to conventional surgical treatment. Int Drug Res Tech 2012;2:411-421.

19. Rosengren A, Faxius L, Tanaka N, Watanabe M, Bjursten LM. Comparison of implantation and cytotoxicity testing for initially toxic biomaterials. J Biomed Mater Res A 2005;75:115-122. doi: 10.1002/jbm.a.30431.

20. Steinberg D, Friedman M, Soskolne A, Sela MN. A new degradable controlled release device for treatment of periodontal disease: in vitro release study. J Periodontol 1990;61:393-398. doi: 10.1902/jop.1990.61.7.393.

21. Hjeljord LG, Rolla G, Bonesvoll P. Chlorhexidine-protein interactions. J Periodontal Res Suppl 1973;12:11-16. doi: 10.1111/j.1600-0765.1973.tb02158.x.

22. Westling M, Tynelius-Bratthall G. Microbiological and clinical short-term effects of repeated intracrevicular chlorhexidine rinsings. J Periodontal Res 1984;19:202-209. doi: 10.1111/j.1600-0765.1984.tb00809.x.

23. Soskolne A, Golomb G, Friedman M, Sela MN. New sus- tained release dosage form of chlorhexidine for dental use. II. Use in periodontal therapy. J Periodontal Res 1983;18:330-336. doi: 10.1111/j.1600-0765.1983. tb00368.x.

24. Stabholz A, Sela MN, Friedman M, Golomb G, Soskolne A. Clinical and microbiological effects of sustained release chlorhexidine in periodontal pockets. J Clin Periodontol 1986;13:783-788. doi: 10.1111/j.1600-051x.1986. tb00882.x.

25. Soskolne WA, Heasman PA, Stabholz A, Smart GJ, Palmer M, Flashner M, Newman HN. Sustained local delivery of chlorhexidine in the treatment of periodontitis: a multicenter study. J Periodontol 1997;68:32-38. doi: 10.1902/ jop.1997.68.1.32.

26. Jeffcoat MK, Bray KS, Ciancio SG, Dentino AR, Fine DH, Gordon JM, Gunsolley JC, Killoy WJ, Lowenguth RA, Magnusson NI, Offenbacher S, Palcanis KG, Proskin HM, Finkelman RD, Flashner M. Adjunctive use of a subgingival controlled-release chlorhexidine chip reduces probing depth and improves attachment level compared with scaling and root planing alone. J Periodontol 1998;69:989-997. doi: 10.1902/jop.1998.69.9.989.

27. Pattnaik S, Anand N, Chandrasekaran SC, Chandrashekar L, Mahalakshmi K, Satpathy A. Clinical and antimicrobial efficacy of a controlled-release device containing chlorhexidine in the treatment of chronic periodontitis. Eur J Clin Microbiol Infect Dis 2015;34:2103-2110. doi: 10.1007/ s10096-015-2459-x.

28. Berman CL, Jaffin RA, Greenstein G. The chlorhexidine question. J Periodontol 1984;55:668-669. doi: 10.1902/ jop.1984.55.11.668.

29. Banting D. Clinical effectiveness of a $0.12 \%$ chlorhexidine mouthrinse over two years. J Dent Res 1989;68:1716-1718.

30. Harvey BV, Squier CA, Hall BK. Effects of chlorhexidine on the structure and permeability of hamster cheek pouch mucosa. J Periodontol 1984;55:608-614. doi: 10.1902/ jop.1984.55.10.608.

31. Fiorentino M, Corrêa FA, Salgado MAN, Regina H. Analytical methods for the determination of chlorhexidine: a review. Crit Rev Anal Chem 2010;40:89-101.

32. Stanley A, Wilson M, Newman HN. The in vitro effects of chlorhexidine on subgingival plaque bacteria. J Clin Periodontol 1989;16:259-264. doi: 10.1111/j.1600-051x.1989. tb01651.x. 